\title{
Atherothrombosis as a systemic, often silent, disease
}

\author{
Valentin Fuster and Pedro R Moreno
}

Atherothrombotic vascular disease (AVD) is a systemic, diffuse condition involving the coronary and peripheral arteries, and is the most common cause of death and disability worldwide. More than 25 million people in the US have at least one clinical manifestation of AVD, but in many more the disease remains a silent harbinger of future cardiovascular events. Although AVD starts in infancy, manifestations are seen in adulthood as coronary artery disease (CAD), stroke and aortic, renal and peripheral vascular disease. Frequently, however, only one territory is symptomatic while the other regions remain clinically silent.

CAD is the most common and devastating manifestation of AVD. In the US alone, the prevalence of acute myocardial infarction is 7.5 million and the incidence 1.25 million, affecting a new patient every $30 \mathrm{~s}$. For stroke, the second most common manifestation, 600,000 new or recurrent strokes occur every year in the US and are the leading cause of AVD-related, long-term disability. Aortic disorders, including abdominal and thoracic aneurysms, aortic dissection and peripheral atheroembolization, comprise the third most common group of manifestations. The prevalence of abdominal aortic aneurysms is $4.7 \%$ of men and $1.7 \%$ of women aged $56-74$ years. The fourth manifestation of AVD is renal artery stenosis, defined as $\geq 1$ renal artery with 50$70 \%$ occlusion, and is reported in $6-8 \%$ of patients undergoing cardiac catheterizations. It has the least understood epidemiology of all the manifestations. Renal artery stenosis is a common cause of recently diagnosed systemic hypertension in patients older than 50 years; this often silent manifestation can be very dangerous.

Lower-extremity peripheral arterial disease (PAD) constitutes the fifth manifestation of

\section{...physicians \\ need to \\ approach all \\ manifestations \\ of AVD, \\ whether \\ clinically \\ symptomatic \\ or silent, as \\ one pathologic \\ entity...}

VF Fuster is

the Editor-in-

Chief of Nature

Clinical Practice

Cardiovascular

Medicine and

PR Moreno is an

Associate Professor

in the Zena and

Michael A. Wiener

Cardiovascular

Institute and The

Marie-Josee and

Henry R. Kravis

Cardiovascular Health

Center, Mount Sinai

School of Medicine,

New York, NY, USA.

\section{Competing interests}

The authors declared

they have no competing

interests.

www.nature.com/clinicalpractice doi:10.1038/ncpcardio0310
AVD. According to the Framingham study, its prevalence has increased from $0.5 \%$ in 1970 to $3.6 \%$ in 2002 . The revascularization rate in symptomatic patients is $5-10 \%$ at 5 years; critical leg ischemia develops in $5 \%$ of these patients, of whom 1-4\% require amputation. Most importantly, the relative risk of death from cardiovascular causes in patients with PAD is about the same as in patients with coronary or cerebrovascular disease. In other words, PAD is a marker or risk for myocardial infarction and stroke in such a patient. As a marker of systemic atherothrombosis, PAD demands a comprehensive clinical approach that fosters prevention, detection and timely intervention. Physicians and health-care providers should use the ankle-brachial index $(A B I)$ to identify PAD. Only an ordinary bloodpressure cuff and a Doppler ultrasound sensor are required. Blood-pressure measurements are taken in the brachial artery in both arms and the left and right posterior tibial arteries and dorsalis pedis arteries. A score of $\leq 0.90$ is $90 \%$ sensitive and $95 \%$ specific for PAD. After diagnosis, the disease can be easily quantified by noninvasive testing. The coronary, the supra-aortic (carotids) and the distal runoff vessels (renal and lower extremities) can be simultaneously assessed with contrastenhanced, coronary and whole-body CT or magnetic resonance angiography. If obstructive CAD is suspected, however, the stress test is needed to stratify risk.

In summary, physicians need to approach all manifestations of AVD, whether clinically symptomatic or silent, as one pathologic entity that intermittently affects different vascular territories. This approach will result in appropriate risk stratification and therapy, reductions in future events, and a better quality of life for our patients. 